\title{
EXISTENCE OF REACTION-DIFFUSION-CONVECTION WAVES IN UNBOUNDED STRIPS
}

\author{
M. BELK, B. KAZMIERCZAK, AND V. VOLPERT \\ Received 30 May 2004 and in revised form 23 November 2004
}

Existence of reaction-diffusion-convection waves in unbounded strips is proved in the case of small Rayleigh numbers. In the bistable case the wave is unique, in the monostable case they exist for all speeds greater than the minimal one. The proof uses the implicit function theorem. Its application is based on the Fredholm property, index, and solvability conditions for elliptic problems in unbounded domains.

\section{Introduction}

Propagation of reaction-diffusion waves, if it occurs in a liquid or in a gaseous medium, can be accompanied by natural convection. Convection can influence the speed and the stability of the front, or can result in its extinction. Influence of natural convection on propagating polymerization fronts is studied in detail experimentally $[1,2,3,4,9]$ and theoretically $[7,8]$ (see also $[6,10,17]$ ) with the use of formal asymptotic expansions. In particular, it is shown how convection changes the onset of thermal instability. Conditions of convective instability of thermal fronts are determined.

Probably the first mathematical work devoted to reaction-diffusion fronts with convection is [19] where it is shown that in some cases the corresponding eigenvalue problem can be reduced to a monotone system and, consequently, a minimax representation for the principal eigenvalue can be obtained $[14,16,20]$. This allows one to obtain conditions of convective instability of reaction-diffusion fronts.

Bifurcations of convective fronts and their stability are studied in [12, 13]. These results concern the upward propagating exothermic fronts in a gaseous medium. The main result can be formulated in a physically clear way: if the Rayleigh number $R$ is sufficiently large, then the reaction-diffusion wave loses its stability, and a reaction-diffusionconvection wave appears. This result is consistent with the experimental observations and with the results of the formal asymptotic analysis, though both of them concern a liquid and not a gaseous medium. 
There is a principal difference between vertically propagating fronts and fronts propagating in any other direction. In the first case, if the Rayleigh number is sufficiently small, then there is no convection. If it is sufficiently large, a convective reaction-diffusion front appears as a result of a bifurcation. This means that the reaction-diffusion front without convection is still a solution of the problem with convection but it is unstable.

If the front is not vertical but propagates in another direction, then the solution without convection exists only for $R=0$. For any $R \neq 0$, a pure reaction-diffusion front cannot exist. Therefore the natural question is as follows: suppose that for $R=0$ there is a reaction-diffusion front. Is there a reaction-diffusion-convection front for $R \neq 0$ and sufficiently small?

An answer to this question could be given by the implicit function theorem. However it is not directly applicable to travelling waves because the linearized problem has a zero eigenvalue. This is also the reason why solutions invariant with respect to translation are not generically structurally stable. A small perturbation of the system can result in disappearance of solutions.

In this work, we show how to apply the implicit function theorem to such problems. It will allow us in particular to prove existence of reaction-diffusion waves with convection. This construction is based on the theory of elliptic problems in unbounded domains: Fredholm property, index, solvability conditions.

We consider the reaction-diffusion equation or system coupled with the Navier-Stokes equations under the Boussinesq approximation in the stream function - vorticity formulation:

$$
\begin{gathered}
u \frac{\partial \theta}{\partial x}+v \frac{\partial \theta}{\partial y}=a \Delta \theta+c \frac{\partial \theta}{\partial x}+F(\theta) \\
u \frac{\partial \omega}{\partial x}+v \frac{\partial \omega}{\partial y}=P \Delta \omega+c \frac{\partial \omega}{\partial x}-R \frac{\partial \theta}{\partial x} \\
\Delta \psi+\omega=0 .
\end{gathered}
$$

Here the components of the vector $\theta$ correspond to the temperature and to the concentrations, $\psi$ is the stream function, $\omega$ the vorticity, $u=\partial \psi / \partial y$ is the horizontal component of the velocity, $v=-\partial \psi / \partial x$ is its vertical component, $x$ is the coordinate along the axis of the $2 \mathrm{D}$ strip, $y$ is the orthogonal variable, $P$ and $R$ are positive parameters. The gravity acts along the $y$-direction.

We consider the no-flux boundary conditions for the temperature and free-surface boundary conditions for the velocity:

$$
y=0, \quad H: \frac{\partial \theta}{\partial y}=0, \quad \omega=0, \quad \psi=0 .
$$

If $R=0$, then (1.2), (1.3) become independent of (1.1). Their solution is $\psi=\omega=0$. Therefore, $u=v=0$, and (1.1) is a usual reaction-diffusion equation describing travelling wave solutions. We assume that $F(0)=F(1)=0$. If the nonlinearity $F$ is bistable, that is, $F^{\prime}(0)<0, F^{\prime}(1)<0$, then, as it is well known, the solution of the problem

$$
\theta^{\prime \prime}+c \theta^{\prime}+F(\theta)=0, \quad \theta(-\infty)=1, \theta(+\infty)=0,
$$


if it exists, is unique up to translation in space. In the monostable case, where $F^{\prime}(0)>0$, $F^{\prime}(1)<0$, the waves are not unique and exist for a half-closed bounded or unbounded interval of wave speeds.

The main result of this work concerns the existence of reaction-diffusion-convection waves for small $R$.

Theorem 1.1. Suppose that in the bistable case problem (1.5) has a solution. Then for all $R$ sufficiently small problem (1.1)-(1.4) has a solution for some $c$ that depends on $R$.

In the monostable case problem (1.1)-(1.4) has a solution for all $R$ sufficiently small and for all $c$ for which problem (1.5) has a solution (see Remark 4.2 at the end of Section 4).

The proof of this theorem is based on the application of the implicit function theorem. For this we need the invertibility of the linearized operator. To prove it we study properties of elliptic operators in unbounded domains. We verify that the operators under consideration satisfy the Fredholm property and compute their index. In the bistable case the index equals zero while the dimension of the kernel of the operator is positive because of the zero eigenvalue related to the invariance of solutions with respect to translation in space. Therefore the codimension of the image of the operator has also a positive dimension. This means that there are some solvability conditions that should be satisfied. We show that these solvability conditions can be formulated in terms of solutions of the homogeneous formally adjoint problem. The explicit form of solvability conditions and some spectral properties of the linearized operator allow us to prove that if the operator is linearized also with respect to the unknown wave speed, then it is invertible, and the implicit function theorem is applicable.

In the monostable case, the index of the operator is positive, and the number of solvability conditions can be zero. In this case we do not need to vary the wave speed to satisfy the solvability conditions. Existence of reaction-diffusion-convection waves in this case is proved for a fixed speed.

The contents of the paper are as follows. In the next section, we recall some results on the existence of waves for reaction-diffusion systems that will be used below. In Section 3, we discuss the structural stability of families of solutions. We show how the Fredholm property of elliptic operators, their index, and solvability conditions allow the application of the implicit function theorem. It is illustrated with some reaction-diffusion problems. In the last section, this approach is used to study existence of reaction-diffusionconvection waves.

\section{Existence of reaction-diffusion waves}

2.1. 1D waves. Consider first the scalar equation

$$
u^{\prime \prime}+c u^{\prime}+F(u)=0
$$

where $F(0)=F(1)=0$ and $F(u) \in C^{1}[0,1]$. In the bistable case, that is, if $F^{\prime}(0)<0$, $F^{\prime}(1)<0$, if the wave with the limits at infinity

$$
u(-\infty)=1, \quad u(+\infty)=0
$$


exists, then it is unique. The wave may exist or not depending on the function $F$. In the simplest case, where

$$
F(u)<0, \quad 0<u<a, \quad F(u)>0, \quad a<u<1
$$

for some $a \in(0,1)$, the wave exists. In the general bistable case, there exists a minimal system of waves that can contain a single wave, a finite, or even infinite number of waves [16].

In the monostable case, where $F^{\prime}(0)>0, F^{\prime}(1)<0$, if a wave exists for some $c$, then waves exist for all values of $c$ from some interval $\left[c_{0}, c_{1}\right)$, where $c_{1}$ can be finite or infinite. In the simplest case, where $F(u)$ is positive for $u \in(0,1)$, the waves exist for all $c \geq c_{0}$. In the general case, waves may not exist. In this case, we should consider systems of waves.

These results are generalized for monotone systems where

$$
\frac{\partial F_{i}}{\partial u_{j}}>0, \quad i, j=1, \ldots, n \text {. }
$$

Let $u=\left(u_{1}, \ldots, u_{n}\right), F=\left(F_{1}, \ldots, F_{n}\right)$. Suppose that $F\left(u_{ \pm}\right)=0$, where $u_{+}<u_{-}$, and the inequality between the vectors are understood component-wise. In the bistable case, we suppose that all eigenvalues of the matrices $F^{\prime}\left(u_{ \pm}\right)$have negative real parts. If in the interval $u_{+}<u<u_{-}$, there are no other stable zeros of the function $F(u)$, then there exists a unique wave with the limits $u(-\infty)=u_{-}, u(+\infty)=u_{+}$. It is monotone with respect to $x$. This result remains valid if the inequality in (2.4) is not strict (under some weaker conditions) and for locally monotone systems where these conditions are imposed only at zero surfaces of the functions $F_{i}$.

If there are other stable zeros in $u_{+}<u<u_{-}$, then, as in the case of the scalar equation, the wave may not exist, and we should consider systems of waves. In the monostable case, without stable intermediate zero, waves exist for $c \geq c_{0}$. Existence of $1 \mathrm{D}$ reaction-diffusion waves for other systems is reviewed in [16].

2.2. Multidimensional waves. We consider the parabolic system of equations

$$
\frac{\partial u}{\partial t}=a\left(x^{\prime}\right) \Delta u+\sum_{j=1}^{m} b_{j}\left(x^{\prime}\right) \frac{\partial u}{\partial x_{j}}+F\left(u, x^{\prime}\right)
$$

in a cylinder $\Omega=\Omega^{\prime} \times R$. Here $u(x)=\left(u_{1}(x), \ldots, u_{n}(x)\right), x=\left(x_{1}, \ldots, x_{m}\right), x_{1}$ is the variable along the axis of the cylinder, $x^{\prime}=\left(x_{2}, \ldots, x_{m}\right)$ is the variable in the section $\Omega^{\prime}$ of the cylinder, the domain $\Omega^{\prime}$ is bounded and has a boundary of the class $C^{2+\delta}$ with some positive $\delta, a\left(x^{\prime}\right), b_{j}\left(x^{\prime}\right)$ are smooth diagonal matrices,

$$
a_{i}\left(x^{\prime}\right) \geq a_{0}>0, \quad x^{\prime} \in \Omega^{\prime}, i=1, \ldots, n, j=1, \ldots, m,
$$

where $a_{i}$ are diagonal elements of the matrix $a, F=\left(F_{1}, \ldots, F_{n}\right)$ is a smooth vector-valued function. On the boundary $\partial \Omega$ of the cylinder we consider the boundary conditions

$$
u(x)=\phi\left(x^{\prime}\right), \quad x \in \partial \Omega,
$$

where $\phi\left(x^{\prime}\right) \in C^{2+\delta}(\bar{\Omega})$ depends only on the variable in the section of the cylinder. 
Travelling wave solution of the problem (2.5), (2.7) is a solution of the form

$$
u(x, t)=w\left(x_{1}-c t, x_{2}, \ldots, x_{m}\right)
$$

where $c$ is an unknown constant, the wave velocity. This function is a solution of the problem

$$
\begin{gathered}
a\left(x^{\prime}\right) \Delta w+c \frac{\partial w}{\partial x_{1}}+\sum_{j=1}^{m} b_{j}\left(x^{\prime}\right) \frac{\partial w}{\partial x_{j}}+F\left(w, x^{\prime}\right)=0, \\
w(x)=\phi\left(x^{\prime}\right), \quad x \in \partial \Omega .
\end{gathered}
$$

We assume that for $n>1$, the nonlinearity $F$ satisfies the following condition:

$$
\frac{\partial F_{i}}{\partial u_{j}} \geq 0, \quad i \neq j, i, j=1, \ldots, n .
$$

This condition means that we can use comparison theorems for the systems under consideration. For $n=1$, there are no additional conditions and the comparison theorems are also applicable. The systems of this type arise in numerous applications (see [16]).

We look for the travelling waves having limits at infinity:

$$
\lim _{x_{1} \rightarrow \pm \infty} w(x)=w_{ \pm}\left(x^{\prime}\right)
$$

where the functions $w_{ \pm}$are solutions of the problem in the section of the cylinder

$$
\begin{gathered}
a\left(x^{\prime}\right) \Delta^{\prime} w_{ \pm}+\sum_{j=2}^{m} b_{j}\left(x^{\prime}\right) \frac{\partial w_{ \pm}}{\partial x_{j}}+F\left(w_{ \pm}, x^{\prime}\right)=0, \\
w_{ \pm}\left(x^{\prime}\right)=\phi\left(x^{\prime}\right), \quad x^{\prime} \in \partial \Omega^{\prime},
\end{gathered}
$$

where $\Delta^{\prime}$ is the Laplace operator with respect to the variables in the section of the cylinder.

We recall the classification of the problems according to the stability of solutions $w_{+}$ and $w_{-}$. We consider the eigenvalue problem for the corresponding linearized equation

$$
\begin{gathered}
L^{ \pm} u \equiv a\left(x^{\prime}\right) \Delta^{\prime} u+\sum_{j=2}^{m} b_{j}\left(x^{\prime}\right) \frac{\partial u}{\partial x_{j}}+F^{\prime}\left(w_{ \pm}\left(x^{\prime}\right), x^{\prime}\right) u=\lambda u, \\
u=0, \quad x^{\prime} \in \partial \Omega^{\prime} .
\end{gathered}
$$

If all eigenvalues of both operators $L^{+}$and $L^{-}$are in the left half-plane, then it is socalled bistable case. If for one of them there are eigenvalues in the right half-plane and for another one all eigenvalues have negative real parts, it is the monostable case. Finally, in the unstable case both operators have eigenvalues in the right half-plane. As it is well known (see [16]), properties of travelling waves are different in these three cases. 
(1) Bistable case. In this section, we consider the problem

$$
\begin{gathered}
a \Delta w+c \frac{\partial w}{\partial x_{1}}+F(w)=0 \\
\left.w\right|_{\partial \Omega}=0 .
\end{gathered}
$$

The constant $c$, the wave velocity, is unknown and should be found together with the function $w$ as a solution of the problem.

We consider travelling waves having limits at infinities:

$$
\lim _{x_{1} \rightarrow \pm \infty} w(x)=w_{ \pm}\left(x^{\prime}\right)
$$

where $w_{+}\left(x^{\prime}\right)$ and $w_{-}\left(x^{\prime}\right)$ are given vector-valued functions,

$$
w_{+}\left(x^{\prime}\right)<w_{-}\left(x^{\prime}\right), \quad x^{\prime} \in G .
$$

The functions $w_{+}\left(x^{\prime}\right)$ and $w_{-}\left(x^{\prime}\right)$ are solutions of the equation in the section of the cylinder

$$
a \Delta^{\prime} v+F(v)=0,\left.\quad v\right|_{\partial G}=0,
$$

where $\Delta^{\prime}$ is the Laplace operator in the section of the cylinder, $\partial G$ is the boundary of $G$.

Theorem 2.1. Let system (2.14) be monotone, let the matrix $F^{\prime}(0)$ be irreducible, and let $w_{+}$and $w_{-}$be stable solutions of (2.18). Suppose that all solutions $v\left(x^{\prime}\right)$ of problem (2.18) satisfying the inequality

$$
w_{+}\left(x^{\prime}\right) \leq v\left(x^{\prime}\right) \leq w_{-}\left(x^{\prime}\right), \quad v\left(x^{\prime}\right) \neq w_{ \pm}\left(x^{\prime}\right)
$$

are unstable. Then there exists a unique monotone in $x_{1}$ travelling wave, that is, a constant $c$ and a classical solution $w(x)$ of (2.14), (2.15) satisfying (2.16).

The proof of this theorem can be found in [15].

The following representation takes place:

$$
c=\inf _{\rho \in K} \sup _{x, i} \frac{a_{i} \Delta \rho_{i}+F_{i}\left(\rho, x^{\prime}\right)}{-\partial \rho / \partial x_{1}}=\sup _{\rho \in K} \inf _{x, i} \frac{a_{i} \Delta \rho_{i}+F_{i}\left(\rho, x^{\prime}\right)}{-\partial \rho / \partial x_{1}} .
$$

Here, $a_{i}$ are the diagonal elements of the matrix $a, F_{i}$ and $\rho_{i}$ are the elements of the vectors $F$ and $\rho$, respectively, $K$ is the class of functions continuous with second derivatives, decreasing in $x_{1}$, satisfying the boundary conditions, and such that

$$
\|\rho(x)-w(x)\|_{L_{2}}<\infty .
$$

In $1 \mathrm{D}$ case, a similar representation was obtained in [16].

Suppose now that there exists a stable solution $w_{0}$ of problem (2.18) satisfying the inequality

$$
w_{+}\left(x^{\prime}\right) \leq w_{0}\left(x^{\prime}\right) \leq w_{-}\left(x^{\prime}\right) .
$$


For simplicity, we restrict ourselves to the case where there is only one such solution, and all other solutions of (2.18) satisfying (2.22), which are not equal to $w_{ \pm}$, are unstable. By Theorem 2.1, there exist a $\left[w_{+}, w_{0}\right]$-wave and a $\left[w_{0}, w_{-}\right]$-wave with the limits at infinities

$$
\begin{array}{ll}
\lim _{x_{1} \rightarrow+\infty} w(x)=w_{+}\left(x^{\prime}\right), & \lim _{x_{1} \rightarrow-\infty} w(x)=w_{0}\left(x^{\prime}\right), \\
\lim _{x_{1} \rightarrow+\infty} w(x)=w_{0}\left(x^{\prime}\right), & \lim _{x_{1} \rightarrow-\infty} w(x)=w_{-}\left(x^{\prime}\right),
\end{array}
$$

respectively. Denote $c_{+}$and $c_{-}$as their velocities.

THeOREM 2.2. Under the assumptions of Theorem 2.1, suppose that there exists one stable solution $w_{0}\left(x^{\prime}\right)$ of (2.18) satisfying (2.19). Then a monotone in $x_{1}$ solution of the problem (2.14)-(2.16) exists if and only if $c_{+}<c_{-}$. Moreover, the velocity $c$ satisfies the inequality $c_{+} \leq c \leq c_{-}$.

The proof of this theorem can be found in [15].

Suppose that $c_{+} \geq c_{-}$. Then it follows from Theorem 2.2 that the wave $\left[w_{+}, w_{-}\right]$does not exist. Similarly to the results in the 1D case [16], we can consider ordered systems of waves.

A system of waves consisting of two waves as above is called ordered if $c_{+} \geq c_{-}$. A similar definition can be given for a system of any finite number of waves. In particular, a system which consists of one wave is ordered by definition.

For the scalar equation, the minimal systems of waves mentioned above are ordered. In the bistable case, the ordered system of waves is unique. The principal difference of system of equations is that the ordered system of waves may be nonunique [18].

Theorem 2.2 implies that if there exists one stable intermediate solution of (2.18), then an ordered system of waves exists and consists of one wave (if $c_{+}<c_{-}$) or of two waves (if $\left.c_{+} \geq c_{-}\right)$. This result may be generalized for the case of a finite number of stable intermediate solutions of (2.18) as it was done in the $1 \mathrm{D}$ case.

(2) Monostable case. We make the following assumptions on the solutions $w_{+}$and $w_{-}$of the problem (2.9), (2.11).

Assumption 2.3. The functions $w_{ \pm}$belong to $C^{(2+\delta)}\left(\bar{\Omega}^{\prime}\right)$ and the following inequality

$$
w_{+}\left(x^{\prime}\right)<w_{-}\left(x^{\prime}\right), \quad x^{\prime} \in \Omega^{\prime}
$$

holds.

Assumption 2.4. There are no other solutions of the problem (2.9), (2.11) satisfying the inequality

$$
w_{+}\left(x^{\prime}\right) \leq w \leq w_{-}\left(x^{\prime}\right), \quad x^{\prime} \in \Omega^{\prime} .
$$

Assumption 2.5. There exists a sequence of functions $\left\{v_{n}\left(x^{\prime}\right)\right\}$ uniformly bounded in $C^{(2+\delta)}\left(\bar{\Omega}^{\prime}\right)$ satisfying the inequality $(2.25)$ and the following conditions:

$$
v_{n}\left(x^{\prime}\right) \longrightarrow w_{+}\left(x^{\prime}\right) \text { as } n \longrightarrow \infty
$$


uniformly in $x^{\prime}$,

$$
\begin{gathered}
a\left(x^{\prime}\right) \Delta^{\prime} v_{n}+\sum_{j=2}^{m} b_{j}\left(x^{\prime}\right) \frac{\partial v_{n}}{\partial x_{j}}+F\left(v_{n}, x^{\prime}\right) \geq 0, \\
v_{n}\left(x^{\prime}\right)=\phi\left(x^{\prime}\right), \quad x^{\prime} \in \partial \Omega^{\prime} .
\end{gathered}
$$

Theorem 2.6. Let Assumptions 2.3, 2.4, and 2.5 be satisfied. Then there exists a constant $c_{0}$ such that for every $c \geq c_{0}$ there exists a monotone in $x_{1}$ solution of the problem (2.5), (2.7). The constant $c_{0}$ is given by the minimax representation

$$
c_{0}=\inf _{\rho \in K} \sup _{x \in \Omega, i=1, \ldots, n} B_{i}
$$

where

$$
B_{i}=\frac{a_{i}\left(x^{\prime}\right) \Delta \rho_{i}+\sum_{j=1}^{m} b_{i j}\left(x^{\prime}\right)\left(\partial \rho_{i} / \partial x_{j}\right)+F_{i}\left(\rho, x^{\prime}\right)}{-\partial \rho_{i} / \partial x_{1}},
$$

$b_{i j}$ are the diagonal elements of the matrix $b_{j}, K$ is a class of sufficiently smooth vector-valued functions $\rho(x)$ such that

$$
\lim _{x_{1} \rightarrow \pm \infty} \rho(x)=w_{ \pm}\left(x^{\prime}\right), \quad \rho(x)=\phi\left(x^{\prime}\right) \text { for } x \in \partial \Omega,
$$

the derivative $\partial \rho / \partial x_{1}$ is negative, and the normal derivative

$$
\frac{\partial\left(\rho-w_{+}(x)\right)}{\partial v}, \quad x \in \partial \Omega
$$

in the direction of the outer normal is also negative. For $c<c_{0}$ such solutions do not exist.

The proof of this theorem can be found in [15].

\section{Structural stability of families of solutions of operator equations}

3.1. Structural instability. Consider an operator $A(u)$ acting from a Banach space $E$ to another Banach space $F$. Suppose that it is continuous, and that it has a Fréchet derivative $A^{\prime}(u) v: E \rightarrow F$ for all $u$ in a neighborhood of some $u_{0} \in E$. We assume that $A^{\prime}(u)$ is a bounded operator that satisfies the Fredholm property. We denote by $\alpha$ the dimension of its kernel, $\beta$ the codimension of its image, $\kappa=\alpha-\beta$ its index. They can depend on the point $u$ about which the operator is linearized.

Let the equation

$$
A(u)=0
$$

have a family of solutions $u(h) \in E$, where $h=\left(h_{1}, \ldots, h_{k}\right)$ is a vector-valued parameter. Suppose next that for any $h$ in a neighborhood of some value $h_{0}$,

$$
u(h)=u\left(h_{0}\right)+\sum_{j=1}^{k} h_{j} v_{j}+o(|h|),
$$


where $v_{j}$ are some elements from $E$, which we assume for simplicity to be different from each other. Then

$$
0=A(u(h))=A\left(u\left(h_{0}\right)\right)+A^{\prime}\left(u\left(h_{0}\right)\right) \sum_{j=1}^{k} h_{j} v_{j}+o(|h|) .
$$

Therefore

$$
A^{\prime}\left(u\left(h_{0}\right)\right) v_{j}=0, \quad j=1, \ldots, k,
$$

that is, the operator $A^{\prime}\left(u\left(h_{0}\right)\right)$ has a zero eigenvalue with the multiplicity greater than or equal to $k$.

We consider next a perturbed problem

$$
A(u)+\epsilon B(u)=0
$$

where $B(u): E \rightarrow F$ is a continuous operator that has a Fréchet derivative. Suppose that (3.5) has a family of solutions $u(h, \epsilon)$ such that

$$
u(h, \epsilon)=u(h)+\epsilon w(h)+o(|\epsilon|)
$$

for some $w(h) \in E$. Substituting (3.6) into (3.5), we obtain

$$
0=A(u(h, \epsilon))+\epsilon B(u(h, \epsilon))=A(u(h))+\epsilon A^{\prime}(u(h)) w(h)+\epsilon B(u(h))+o(|\epsilon|) .
$$

Hence

$$
A^{\prime}(u(h)) w(h)=-B(u(h))
$$

This means that the equation

$$
A^{\prime}(u(h)) w=-B(u(h))
$$

has a solution. On the other hand, since we assume that the operator $A^{\prime}(u(h))$ satisfies the Fredholm property, it is solvable if and only if

$$
\phi_{j}(B(u(h)))=0, \quad j=1, \ldots, \beta,
$$

where $\phi_{j}$ are some linearly independent functionals from the space $F^{*}$ dual to $F$. For a given index $\kappa$ of the operator, $\beta=\alpha-\kappa \geq k-\kappa$. Therefore if

$$
\kappa<k \text {, }
$$

then the number of solvability conditions is positive. We can chose an operator $B$ such that the solvability conditions (3.10) are not satisfied. Hence the assumption about the existence of the family of solutions $u(h, \epsilon)$ leads to contradiction. In other words, we obtain 
that a small perturbation of the operator can lead to disappearance of the family of solutions. Thus, family of solutions of operator equations are not generally structurally stable.

Condition (3.11) means that the dimension of the manifold of solutions is greater than the index of the operator. On the other hand, it is well known [11] that the dimension of the manifold of solutions of an operator equation $A(u)=f$ with a Fredholm operator $A$ equals the index of the operator if $f$ is a regular point. Hence (3.11) corresponds to the case where 0 is not a regular point of the operator $A$.

Example 3.1. Consider the operator

$$
A(u)=u^{\prime \prime}+F(u)
$$

acting from $C^{2+\delta}(R)$ to $C^{\delta}(R)$. Let the function $F(u)$ satisfy the following conditions:

$$
F(0)=0, \quad F^{\prime}(0)<0, \quad \int_{0}^{1} F(u) d u=0, \quad \int_{0}^{u} F(s) d s<0, \quad 0<u<1 .
$$

Then there exists a solution $u_{0}(x)$ of the problem

$$
u^{\prime \prime}+F(u)=0, \quad u( \pm \infty)=0 .
$$

It is invariant with respect to translation: any function $u_{0}(x+h), h \in R$ is also a solution. Therefore there exists a one-parameter family of solutions. The index of the linearized operator $A^{\prime}\left(u_{0}\right)$ equals zero [5]. Thus, the dimension of the family of solutions is greater than the index.

The linearized operator

$$
A^{\prime}\left(u_{0}\right)=u^{\prime \prime}+F^{\prime}\left(u_{0}\right) u
$$

is selfadjoint. The eigenfunction of the formally adjoint operator $\left(A^{\prime}\left(u_{0}\right)\right)^{*}=A^{\prime}\left(u_{0}\right)$ corresponding to the zero eigenvalue coincides with the eigenfunction of the operator $A^{\prime}\left(u_{0}\right)$ corresponding to the zero eigenvalue, that is, with $u_{0}^{\prime}(x)$. Therefore the equation

$$
u^{\prime \prime}+F^{\prime}\left(u_{0}\right) u=f
$$

is solvable if and only if

$$
\int_{-\infty}^{\infty} f(x) u_{0}^{\prime}(x) d x=0
$$

However, the function $u_{0}^{\prime}(x)$ is odd. Therefore for any $g(u)$,

$$
\int_{-\infty}^{\infty} g\left(u_{0}(x)\right) u_{0}^{\prime}(x) d x=0
$$

and the solvability condition is satisfied.

This does not prove yet that the family of solutions persists under a perturbation of the nonlinearity because we have obtained the solvability condition as a necessary condition. 
However it can be easily proved directly that if a perturbation is sufficiently small, then a one-parameter family of solutions still exists.

This example is not in contradiction with the general construction described above. In this particular case, any autonomous perturbation satisfies the solvability condition. A nonautonomous perturbation may not satisfy it.

Example 3.2. Consider the same problem as above except that we assume in addition that

$$
F(u)<0, \quad 0<u<\frac{1}{2}, \quad F(u)>0, \quad \frac{1}{2}<u<1,
$$

and that the limits of the solution at infinity are different:

$$
u(-\infty)=1, \quad u(\infty)=0 .
$$

It is well known (see [16]) that this problem has a solution if and only if

$$
\int_{0}^{1} F(u) d u=0 .
$$

Therefore a small perturbation of $F$ such that the integral becomes different from zero leads to disappearance of the family of solutions.

3.2. Problems with parameter. Consider an operator $A(u, c, \epsilon)$ depending on a vectorvalued parameter $c=\left(c_{1}, \ldots, c_{m}\right)$ and on a parameter $\epsilon$. It is defined on the space $E_{2}=$ $E_{1} \times R$, where $E_{1}=E \times R^{m}$, with the image in $F$. We assume that the operator $A$ is continuous with respect to $(u, c, \epsilon)$.

We will find conditions that would allow us to apply the implicit function theorem. Suppose that the equation

$$
A(u, c, 0)=0
$$

has a family of solutions $\left(u_{0}(h), c^{0}\right)$, where $h=\left(h_{1}, \ldots, h_{k}\right)$ is a vector-valued parameter, and $c^{0}=\left(c_{1}^{0}, \ldots, c_{m}^{0}\right)$ is a constant (independent of $h$ ) vector. We assume that the family of solutions $u_{0}(h)$ admits the representation (3.2) in a neighborhood of each $h^{0}$. As above we suppose that the elements $v_{j}, j=1, \ldots, k$ are different from each other.

Suppose next that there exists a Fréchet derivative $A^{\prime}(u, c, \epsilon): E_{1} \rightarrow F$ of the operator $A$ with respect to the variables $(u, c)$ in a neighborhood of the point $\left(u_{0}\left(h^{0}\right), c^{0}, 0\right)$. We suppose that it is a bounded operator continuous with respect to $(u, c, \epsilon)$ in the operator norm.

To apply the implicit function theorem, we should study the invertibility of the operator $L=A^{\prime}\left(u\left(h^{0}\right), c^{0}, 0\right)$. We have

$$
L(\tilde{u}, \tilde{c})=A_{u}^{\prime}\left(u\left(h^{0}\right), c^{0}, 0\right) \tilde{u}+\sum_{j=1}^{m} A_{c_{j}}^{\prime}\left(u\left(h^{0}\right), c^{0}, 0\right) \tilde{c}_{j},
$$

where $A_{u}^{\prime}$ and $A_{c_{j}}^{\prime}$ are the partial Fréchet derivatives with respect to $u$ and $c_{j}$, respectively. We suppose that the operator $A_{u}^{\prime}\left(u\left(h^{0}\right), c^{0}, 0\right)$ satisfies the Fredholm property with $\alpha$ being 
the dimension of its kernel, $\beta$ the codimension of the image, and $\kappa$ its index. We will discuss the solvability conditions for the equation

$$
L(\tilde{u}, \tilde{c})=f
$$

assuming that $\alpha$ and $\kappa$ are given. We rewrite this equation in the form

$$
A_{u}^{\prime}\left(u\left(h^{0}\right), c^{0}, 0\right) \tilde{u}=f-\sum_{j=1}^{m} A_{c_{j}}^{\prime}\left(u\left(h^{0}\right), c^{0}, 0\right) \tilde{c}_{j} .
$$

We consider below some cases interesting for applications.

Case $1(\kappa=0, \alpha=k)$. Here $\beta=k$. Denote by $\phi_{j} \in F^{*}, j=1, \ldots, k$ linear independent functionals that vanish at the image of the operator $A_{u}^{\prime}\left(u\left(h^{0}\right), c^{0}, 0\right)$. Then (3.25) is solvable if and only if

$$
\sum_{j=1}^{m} \phi_{i}\left(A_{c_{j}}^{\prime}\left(u\left(h^{0}\right), c^{0}, 0\right)\right) \tilde{c}_{j}=\phi_{i}(f), \quad i=1, \ldots, k .
$$

This system is solvable with respect to $\tilde{c}_{j}$ for any $f$ if and only if the matrix $P_{i j}=$ $\phi_{i}\left(A_{c_{j}}^{\prime}\left(u\left(h^{0}\right), c^{0}, 0\right)\right)$ has the rank $k$. We suppose that this condition is satisfied. Then (3.24) is solvable for any $f \in F$. Moreover, it has a $k$-dimensional family of solutions

$$
\tilde{u}=\tilde{u}_{0}+\sum_{j=1}^{k} \tau_{j} v_{j}
$$

where $\tau_{j}$ are constants and $\tilde{c}$ is fixed.

Denote by $\psi_{j}, j=1, \ldots, k$ linearly independent functionals from $E^{*}$ such that

$$
\psi_{j}\left(u_{0}\left(h^{0}\right)\right)=0, \quad j=1, \ldots, k
$$

and the matrix $Q_{i j}=\psi_{j}\left(v_{i}\right)$ is invertible. Let $E_{1}^{0}$ be a subspace of $E_{1}$ such that

$$
\psi_{j}(\tilde{u})=0, \quad j=1, \ldots, k, \forall(\tilde{u}, \tilde{c}) \in E_{1}^{0} .
$$

Then the restriction $L^{0}$ of the operator $L$ to the subspace $E_{1}^{0}$ is invertible. Therefore we can apply the implicit function theorem for the restriction $A^{0}(u, c, \epsilon)$ of the operator $A(u, c, \epsilon)$ :

$$
A^{0}(u, c, \epsilon): E_{1}^{0} \longrightarrow F
$$

Thus we have proved the following theorem.

Theorem 3.3. Suppose that the operator $A(u, c, \epsilon): E_{2} \rightarrow F$ is continuous, and there exists a $k$-dimensional manifold of solutions $\left(u_{0}(h), c^{0}\right)$ of the equation $A(u, c, 0)=0$. 
Assume that the Fréchet derivative $A^{\prime}(u, c, \epsilon): E_{1} \rightarrow F$ exists and satisfies the following properties:

(1) it is a bounded operator, continuous with respect to $(u, c, \epsilon)$ in the operator norm in a neighborhood of $\left(u_{0}\left(h^{0}\right), c^{0}, 0\right)$,

(2) $A_{u}^{\prime}\left(u_{0}\left(h^{0}\right), c^{0}, 0\right)$ is a Fredholm operator with the index zero and with the dimension $k$ of the kernel,

(3) the matrix $P_{i j}=\phi_{j}\left(A_{c_{i}}^{\prime}\left(u_{0}\left(h^{0}\right), c^{0}, 0\right)\right)$, where $\phi_{j}$ are linearly independent functionals vanishing on the image of the operator $A_{u}^{\prime}$, has the rank $k$.

Then for all $\epsilon$ sufficiently small, there exists a unique solution $\left(u_{\epsilon}\left(h^{0}\right), c^{\epsilon}\right)$ of the equation $A(u, c, \epsilon)=0$ satisfying the conditions

$$
\psi_{j}\left(u_{\epsilon}\left(h^{0}\right)\right)=0, \quad j=1, \ldots, k
$$

where $\psi_{j}$ are linearly independent functionals that vanish at the subspace of E complementary to the kernel of the operator $A_{u}^{\prime}$. The family of solutions $\left(u_{\epsilon}\left(h^{0}\right), c^{\epsilon}\right)$ is continuous with respect to $\epsilon$.

This theorem provides structural stability of the family of solutions for an arbitrary but fixed $h^{0}$. This means in particular that the perturbation of the operator can depend on $h^{0}$. In this case, $c^{\epsilon}$ may also depend on $h^{0}$. Therefore we obtain a manifold $\left(u_{\epsilon}\left(h^{0}\right), c^{\epsilon}\left(h^{0}\right)\right)$ of solutions in $E_{1}$. It lies in the neighbourhood of the manifold $\left(u_{0}\left(h^{0}\right), c^{0}\left(h^{0}\right)\right)$, and it does not generally belong to a subspace $c=$ const.

Another conclusion from Theorem 3.3 is that to provide structural stability of $k$ dimensional manifold of solutions, we need $k$ free parameters, that is, $k$ parameters that are not a priori given and that are chosen to solve the problem.

If we look for a solution of the equation

$$
A(u, c, \epsilon)=0
$$

for a given $c$, then we should consider the equation

$$
c^{\epsilon}(h)=c .
$$

Let

$$
c_{\max }=\sup _{h} c^{\epsilon}(h), \quad c_{\min }=\inf _{h} c^{\epsilon}(h) .
$$

Then for any $c_{\min }<c<c_{\max }$ there exists a solution of (3.33) and, consequently, of (3.32). Since $c_{\max }$ and $c_{\min }$ are continuous functions of $\epsilon$, then solutions of (3.33) persist under a small perturbation, though the corresponding value of $h$ is not necessarily continuous with respect to $\epsilon$.

If the perturbation does not depend on $h$, then $c_{\max }=c_{\min }$. Case $2(\kappa=k, \alpha=k)$. Here $\beta=0$. The equation

$$
A_{u}^{\prime}\left(u\left(h^{0}\right), c^{0}, 0\right) \tilde{u}=f
$$


is solvable for any $f \in F$. Therefore we do not need to linearize the operator $A(u, c, \epsilon)$ with respect to $c$. We consider it as acting from $E$ to $F$ for $c^{0}$ fixed.

We have the following theorem.

Theorem 3.4. Suppose that the operator $A(u, c, \epsilon): E \rightarrow F$ is continuous, and there exists a $k$-dimensional manifold of solutions $\left(u_{0}(h), c^{0}\right)$ of the equation $A(u, c, 0)=0$.

Assume that the Fréchet derivative $A_{u}^{\prime}\left(u, c^{0}, \epsilon\right): E \rightarrow F$ exists and satisfies the following properties:

(1) it is a bounded operator, continuous with respect to $(u, \epsilon)$ in the operator norm in a neighborhood of $\left(u_{0}\left(h^{0}\right), 0\right)$,

(2) $A_{u}^{\prime}\left(u_{0}\left(h^{0}\right), 0\right)$ is a Fredholm operator with the index and with the dimension of the kernel equal to $k$.

Then for all $\epsilon$ sufficiently small there exists a unique solution $u_{\epsilon}\left(h^{0}\right)$ of the equation $A\left(u, c^{0}\right.$, $\epsilon)=0$ satisfying the conditions $\psi_{j}\left(u_{\epsilon}\left(h^{0}\right)\right)=0, j=1, \ldots, k$, where $\psi_{j}$ are linearly independent functionals that vanish at the subspace of E complementary to the kernel of the operator $A_{u}^{\prime}$. The family of solutions $u_{\epsilon}\left(h^{0}\right)$ is continuous with respect to $\epsilon$.

Let $c=\left(c_{1}, \ldots, c_{m}\right)$ be as above a vector-valued free parameter. Consider the operator $L=A^{\prime}(u, c, \epsilon)$ linearized with respect to both $u$ and $c$. We write the equation $L(\tilde{u}, \tilde{c})=0$ in the form

$$
A_{u}^{\prime}\left(u\left(h^{0}\right), c^{0}, 0\right) \tilde{u}=-\sum_{j=1}^{m} A_{c_{j}}^{\prime}\left(u\left(h^{0}\right), c^{0}, 0\right) \tilde{c}_{j} .
$$

By virtue of the assumptions above this equation is solvable for any right-hand side. Suppose that the elements $A_{c_{j}}^{\prime}\left(u\left(h^{0}\right), c^{0}, 0\right), j=1, \ldots, m$ are linearly independent. Then the solutions $w_{j}$ of the equations

$$
A_{u}^{\prime}\left(u\left(h^{0}\right), c^{0}, 0\right) \tilde{u}=-A_{c_{j}}^{\prime}\left(u\left(h^{0}\right), c^{0}, 0\right)
$$

are also linearly independent. Moreover, they are linearly independent with the eigenfunctions $v_{i}$ corresponding to the zero eigenvalue of the operator $A_{u}^{\prime}\left(u\left(h^{0}\right), c^{0}, 0\right)$. Indeed, if there is a nontrivial linearly combination

$$
a_{1} v_{1}+\cdots+a_{k} v_{k}+b_{1} w_{1}+\cdots+b_{m} w_{m}=0,
$$

then applying to it the operator $A_{u}^{\prime}\left(u\left(h^{0}\right), c^{0}, 0\right)$ we obtain a contradiction with the assumption that $A_{c_{j}}^{\prime}\left(u\left(h^{0}\right), c^{0}, 0\right)$ are linearly independent.

Thus the dimension of the kernel of the operator $L$ becomes $k+m$. The codimension of its image remains zero. On the other hand, it is surjective. Therefore, 0 is a regular point of the operator $A(u, c, \epsilon)$. Hence the dimension of the manifold of solutions of the equation $A(u, c, \epsilon)=0$ in the space $E_{1}$ equals $k+m$. The dimension $k$ corresponds to a fixed value of the parameter $c$, and additional $m$ dimensions come from $m$ free parameters.

Case $3(\kappa=k, \alpha>k)$. Here $\beta>0$. This case is close to the Case 1 We need free parameters to satisfy the solvability conditions. 
3.3. $1 \mathrm{D}$ reaction-diffusion problems. Consider the $1 \mathrm{D}$ reaction-diffusion system

$$
a u^{\prime \prime}+c u^{\prime}+F(u)=0 .
$$

Here $a$ is a constant matrix, $c$ is a constant. Problems of this type describe travelling wave solutions of parabolic systems. Suppose that for some $c=c_{0}$ there exists a solution of this system with the limits at infinity

$$
\lim _{x \rightarrow \pm \infty} u(x)=u_{ \pm}
$$

The solution of this problem is invariant with respect to translation in space. Therefore there exists a $1 \mathrm{D}$ family of solutions $u_{0}(h)$.

Consider the operator

$$
A(u)=a\left(u+u_{0}\right)^{\prime \prime}+c\left(u+u_{0}\right)^{\prime}+F\left(u+u_{0}\right)
$$

acting from $C_{0}^{2+\delta}(R)$ to $C_{0}^{\delta}(R)$. Here $u_{0}$ is a solution of the problem (3.39)-(3.40) for some given value of $h, C_{0}^{k+\delta}(R)$ denotes the space of functions equal to zero at infinity.

The essential spectrum of the operator $A^{\prime}$, that is, the set of complex $\lambda$ where the operator $A^{\prime}-\lambda$ does not satisfy the Fredholm property is given by the algebraic equation

$$
\operatorname{det}\left(-a \xi^{2}+c i \xi+F^{\prime}\left(u_{ \pm}\right)-\lambda E\right)=0, \quad \xi \in R \text {. }
$$

Here $E$ is the identity matrix.

(1) Bistable case. Suppose that (3.42) does not have solutions for any nonnegative real $\lambda$. Then the operator $A^{\prime}$ is Fredholm with the zero index. It has a zero eigenvalue. If it is simple, then the linearized system

$$
a u^{\prime \prime}+c u^{\prime}+F^{\prime}\left(u_{0}(x)\right) u=f
$$

has a solution if and only if

$$
\int_{-\infty}^{\infty} f(x) v(x) d x=0
$$

where $v(x)$ is a solution of the homogeneous formally adjoint problem

$$
a^{T} v^{\prime \prime}-c v^{\prime}+\left(F^{\prime}\left(u_{0}(x)\right)\right)^{T} v=0 .
$$

Here the superscript $T$ denotes the transposed matrix. Therefore the problem linearized with respect $u$ and $c$,

$$
a u^{\prime \prime}+c_{0} u^{\prime}+F^{\prime}\left(u_{0}(x)\right) u=f-c u_{0}^{\prime}
$$

is solvable for any $f$. Indeed,

$$
\int_{-\infty}^{\infty} u_{0}^{\prime} v(x) d x \neq 0
$$


by virtue of the simplicity of the zero eigenvalue, and $c$ can be chosen to satisfy the solvability condition.

Consider the perturbed operator

$$
A_{\epsilon}(u)=a\left(u^{\prime \prime}+u_{0}^{\prime \prime}\right)+c\left(u^{\prime}+u_{0}^{\prime}\right)+F\left(u+u_{0}\right)+\epsilon g(u, x),
$$

where $g(0, x)=0$. Then $A_{0}(0)=0$. In the space $E=C^{2+\delta}(R)$ we define a subspace $E_{0}=$ $\{u \in E, \phi(u)=0\}$, where $\phi$ is a functional that is different from zero at the eigenfunction $u_{0}^{\prime}$ corresponding to the zero eigenvalue of the linearized operator $A^{\prime}$. We can put, for example,

$$
\phi(u)=\int_{-\infty}^{\infty} u u_{0}^{\prime} d x \quad \text { or } \quad \phi(u)=u_{1}(0)
$$

where $u_{1}$ denotes the first component of the vector $u$. In the second case we take into account that $u_{0}^{\prime}(x) \neq 0, x \in R$. Applying the implicit function theorem we obtain that the equation

$$
A_{\epsilon}(u)=0
$$

has a family of solutions $\left(u_{h}, c_{h}\right)$ for all $\epsilon$ sufficiently small. For the scalar equation and for the monotone systems the principle eigenvalue is simple, and condition (3.47) is satisfied. Otherwise it should be assumed.

(2) Monostable case. In the monostable case, the index of the linearized operator is positive. If the codimension $\beta$ of its image equals zero, then the problem

$$
a u^{\prime \prime}+c_{0} u^{\prime}+F^{\prime}\left(u_{0}(x)\right) u=f
$$

is solvable for any $f$. Therefore perturbed problem (3.48) has a family of solutions $u_{h}$ for $c=c_{0}$ and all $\epsilon$ sufficiently small. For the scalar equation, it can be verified that $\beta=0$. For systems of equations it is not known whether $\beta=0$ or it can be positive. The latter case seems to be not very probable. If $\beta=1$, then the solvability condition can be satisfied by linearization with respect to $c$. For $\beta>1$, it cannot be done.

We note that if $\alpha>0$ and $\beta=0$, then the dimension of the root space is necessarily greater than the dimension of the kernel. Indeed, if $u_{0}$ belong to the kernel of an operator $L$, then $L u_{0}=0$. On the other hand, equation $L u=u_{0}$ has a nonzero solution. Therefore, the equation $L^{2} u=0$ has a nonzero solution different from $u_{0}$.

(3) Reduction of systems. Here, we consider the situation where we reduce the system to the scalar equation or to a system with less equations. We will restrict ourselves to the system of two equations

$$
\begin{gathered}
u^{\prime \prime}+c u^{\prime}+F(u)+g(u, v)=0, \\
v^{\prime \prime}+c v^{\prime}-a v+\epsilon h(u, v)=0 .
\end{gathered}
$$

Suppose that $g(u, 0)=0, a \neq 0$. Then for $\epsilon=0, v=0$ is the solution of the second equation, and the first equation depends only on $u$. 
If the nonlinearity $F$ corresponds to the bistable case, and there exists a solution $u_{0}(x)$ of the equation

$$
u^{\prime \prime}+c_{0} u^{\prime}+F(u)=0
$$

then for $\epsilon$ sufficiently small there exists a solution of system (3.52) close to $\left(u_{0}(x), 0\right)$. This follows from the solvability of the linearized system

$$
\begin{gathered}
u^{\prime \prime}+c_{0} u^{\prime}+F^{\prime}\left(u_{0}(x)\right) u+g_{u}^{\prime}\left(u_{0}(x), 0\right) u+g_{v}^{\prime}\left(u_{0}(x), 0\right) v=f_{1}-c u_{0}^{\prime}(x), \\
v^{\prime \prime}+c_{0} v^{\prime}-a v=f_{2} .
\end{gathered}
$$

We use here the fact that the second component of the solution for $\epsilon=0$ equals zero, and $g_{u}^{\prime}\left(u_{0}(x), 0\right)=0$. Then the second equation in the linearized system does not contain $c$, and it is solvable for any $f_{2}$. From the first equation we find $c$ to satisfy the solvability condition. It is solvable for any $f_{1}$.

The same approach may be not applicable for the system

$$
\begin{gathered}
u^{\prime \prime}+c u^{\prime}+F(u)+\epsilon g(u, v)=0 \\
v^{\prime \prime}+c v^{\prime}+F(v)+\epsilon h(u, v)=0
\end{gathered}
$$

For $\epsilon=0$ the linearized system has the form

$$
\begin{aligned}
u^{\prime \prime}+c_{0} u^{\prime}+F^{\prime}\left(u_{0}(x)\right) u & =f_{1}-c u_{0}^{\prime}(x), \\
v^{\prime \prime}+c_{0} v^{\prime}+F^{\prime}\left(v_{0}(x)\right) v & =f_{2}-c v_{0}^{\prime}(x) .
\end{aligned}
$$

We have only one free parameter $c$ to satisfy two solvability conditions. Therefore the linearized system is not solvable for any $f_{1}$ and $f_{2}$. It can be verified that perturbed system (3.55) may not have solutions.

In the monostable case, we do need to linearize the operator with respect to $c$ :

$$
\begin{gathered}
u^{\prime \prime}+c_{0} u^{\prime}+F^{\prime}\left(u_{0}(x)\right) u=f_{1}, \\
v^{\prime \prime}+c_{0} v^{\prime}+F^{\prime}\left(v_{0}(x)\right) v=f_{2} .
\end{gathered}
$$

The solvability conditions are satisfied. Therefore system (3.55) has solutions for $c=c_{0}$ and $\epsilon$ sufficiently small.

In the mixed case,

$$
\begin{aligned}
& u^{\prime \prime}+c u^{\prime}+F(u)+\epsilon g(u, v)=0 \\
& v^{\prime \prime}+c v^{\prime}+G(v)+\epsilon h(u, v)=0
\end{aligned}
$$

where the nonlinearity $F$ is bistable and $G$ is monostable, the linearized system

$$
\begin{aligned}
u^{\prime \prime}+c_{0} u^{\prime}+F^{\prime}\left(u_{0}(x)\right) u & =f_{1}-c u_{0}^{\prime}(x), \\
v^{\prime \prime}+c_{0} v^{\prime}+G^{\prime}\left(v_{0}(x)\right) v & =f_{2}-c v_{0}^{\prime}(x)
\end{aligned}
$$

is solvable for any $f_{1}$ and $f_{2}$. Indeed, we first choose $c$ to satisfy the solvability condition in the first equation. The second equation is solvable for any right-hand side. Therefore the perturbed system has a solution with a variable $c$. 


\section{Existence of reaction-diffusion-convection waves}

In this section, we study existence of convective fronts described by reaction-diffusionconvection system of equations in unbounded horizontal layers. We will see below that this case is essentially different in comparison with the case of vertically propagating fronts studied in $[12,13]$. We consider the model where a reaction-diffusion system is coupled with the Navier-Stokes equations written in the stream function-vorticity formulation:

$$
\begin{aligned}
\frac{\partial T}{\partial t}+v \cdot \nabla T & =\kappa \Delta T+F(T) \\
\frac{\partial \omega}{\partial t}+v \cdot \nabla \omega & =P \Delta \omega-P R \frac{\partial T}{\partial x} \\
-\Delta \Psi & =\omega .
\end{aligned}
$$

Here $T$ is the temperature of the mixture, $\Psi$ is the stream function, and $\omega$ the vorticity; $\kappa$ is the thermal diffusivity, $P$ is the Prandtl number, and $R$ is the Rayleigh number describing the intensity of free convection. The velocity $v=\left(v_{x}, v_{z}\right)$ is defined as

$$
v_{x}=\frac{\partial \Psi}{\partial z}, \quad v_{z}=-\frac{\partial \Psi}{\partial x} .
$$

This system of equations is considered in the domain

$$
\Omega=\{-\infty<x<+\infty, 0<z<l\}
$$

with the boundary conditions

$$
z=0, \quad l: \frac{\partial T}{\partial z}=0
$$

The free-surface boundary condition for the velocity can be written in terms of the stream function and vorticity as follows:

$$
z=0, \quad l: \omega=0, \quad \Psi=0 .
$$

4.1. Bistable case. Existence and uniqueness of waves depend on stability properties of zeros of the source term $F$. In the bistable case both points $u=0$ and $u=1$ are stable stationary points of the equation

$$
\frac{d u}{d t}=F(u)
$$

The equation

$$
\frac{\partial T}{\partial t}=\kappa \Delta T+F(T)
$$

has a stationary travelling wave solution that has the form

$$
T(x, z, t)=\theta(x-c t)
$$


where $c$ is an unknown wave velocity. Because of the Neumann boundary condition it can be a $1 \mathrm{D}$ planar wave. This solution has the limits at infinity:

$$
\theta_{ \pm}=\lim _{x \rightarrow \pm \infty} \theta(x)
$$

where $F\left(\theta_{ \pm}\right)=0$.

The function $\theta(x)$ satisfies the equation

$$
\kappa \theta^{\prime \prime}+c \theta^{\prime}+F(\theta)=0 .
$$

Moreover this solution is also a travelling wave solution of system of equations (4.1) with $v=0$ and $R=0$. In a vertical layer, this stationary solution exists and is stable as long as $R<R_{\text {critical }}$; then a supercritical bifurcation occurs and this solution is no more stable while a stable convective travelling wave solution bifurcates. In this case, the principal real eigenvalue is negative when $R<R_{\text {critical }}$ and it becomes positive when $R$ passes its critical value.

However there exist no stationary travelling wave solutions for problem (4.1)-(4.5) with $v=0$ and $R \neq 0$ if the domain is not vertical. This makes the main difference between this work and the vertical domain case. The transition from the nonconvective solution for $R=0$ to a convective solution for $R \neq 0$ does not occur through a bifurcation. In fact, it is the same branch of solutions.

A travelling wave solution of problem (4.1)-(4.5) is a solution of the form

$$
T(x, z, t)=T(x-c t, z), \quad \omega(x, z, t)=\omega(x-c t, z), \quad \Psi(x, z, t)=\Psi(x-c t, z) .
$$

It satisfies the system of equations

$$
\begin{gathered}
\kappa \Delta T+\left(c-\frac{\partial \Psi}{\partial z}\right) \frac{\partial T}{\partial x}+\frac{\partial \Psi}{\partial x} \frac{\partial T}{\partial z}+F(T)=0, \\
P \Delta \omega+\left(c-\frac{\partial \Psi}{\partial z}\right) \frac{\partial \omega}{\partial x}+\frac{\partial \Psi}{\partial x} \frac{\partial \omega}{\partial z}+P R \frac{\partial T}{\partial x}=0, \\
\Delta \Psi+\omega=0
\end{gathered}
$$

with the boundary conditions

$$
\frac{\partial T}{\partial z}=0, \quad \omega=0, \quad \Psi=0: z=0, l .
$$

We introduce the spaces of functions

$$
\begin{aligned}
& E_{1}=\left\{T \in C^{(2+\delta)}(\bar{\Omega}), \frac{\partial T}{\partial z}=0: z=0, l\right\}, \\
& E_{2}=\left\{\omega \in C^{(2+\delta)}(\bar{\Omega}), \omega=0: z=0, l\right\}, \\
& E_{3}=\left\{\Psi \in C^{(2+\delta)}(\bar{\Omega}), \Psi=0: z=0, l\right\} .
\end{aligned}
$$


To get rid of the invariance of solutions with respect to translation in space we consider the subspace $E_{1}^{\Theta}$ of the space $E_{1}$ :

$$
E_{1}^{\Theta}=\left\{\Theta \in E_{1}, \Theta\left(x_{0}\right)=0\right\} .
$$

A function $\phi \in C^{(2+\delta)}(\bar{\Omega})$ such that $\lim _{x \rightarrow \pm \infty} \phi=\theta_{ \pm}$and $\phi\left(x_{0}\right)=\left(\theta_{+}+\theta_{-}\right) / 2$ is introduced to work in a space of functions that equal zero at infinity. We can put $\phi=\theta$, where $\theta(x)$ is the solution of (4.10), and represent $T$ as $T=\phi+\Theta$, where $\Theta \in E_{1}^{\Theta}$.

Then we define the product spaces $E=E_{1} \times E_{2} \times E_{3}$ and $E_{\Theta}=E_{1}^{\Theta} \times E_{2} \times E_{3}$. Hence we consider the operator $A: Y \times X \rightarrow Z$ with $X=\mathbb{R}, Y=E_{\Theta} \times \mathbb{R}$, and $Z=\left(C^{(\delta)}(\bar{\Omega})\right)^{3}$ :

$$
A_{R}(u, c)=\left\{\begin{array}{l}
\kappa \Delta(\Theta+\phi)+\left(c-\frac{\partial \Psi}{\partial z}\right) \frac{\partial(\Theta+\phi)}{\partial x}+\frac{\partial \Psi}{\partial x} \frac{\partial(\Theta+\phi)}{\partial z}+F(\Theta+\phi), \\
P \Delta \omega+\left(c-\frac{\partial \Psi}{\partial z}\right) \frac{\partial \omega}{\partial x}+\frac{\partial \Psi}{\partial x} \frac{\partial \omega}{\partial z}+P R \frac{\partial(\Theta+\phi)}{\partial x} \\
\Delta \Psi+\omega .
\end{array}\right.
$$

Thus the problem can be written as

$$
A_{R}(u, c)=0
$$

and $A_{0}\left(0, c_{0}\right)=0$.

The operator $A_{R}(u, c)$ is continuous. The linearized operator $A_{R}^{\prime}\left(u_{0}, c_{0}\right)(u, c)$ is bounded and continuous in the operator norm with respect to $R, u_{0}$, and $c_{0}$.

We consider now the operator linearized with respect to $(u, c)$ about the solution of system without convection, that is, $u_{0}=(\theta(x), 0,0) \in E, c_{0} \in \mathbb{R}$, and $R=0$ :

$$
L(u, c)=\left\{\begin{array}{l}
\kappa \Delta T+c_{0} \frac{\partial T}{\partial x}+c \theta^{\prime}-\frac{\partial \Psi}{\partial z} \theta^{\prime}+F^{\prime}(\theta) T \\
P \Delta \omega+c_{0} \frac{\partial \omega}{\partial x} \\
\Delta \Psi+\omega,
\end{array}\right.
$$

acting from $E \times R$ to $Z$. It should be shown that it satisfies the Fredholm property, that its index equals zero, and that the kernel is empty. Then its invertibility in the subspace $E_{\Theta} \times \mathbb{R}$ will follow.

We introduce the corresponding limiting operator $L_{ \pm}: E \rightarrow Z$ to study the Fredholm property of the operator $L$ :

$$
L_{ \pm}(u, c)=\left\{\begin{array}{l}
\kappa \Delta T+c_{0} \frac{\partial T}{\partial x}+F^{\prime}\left(\theta_{ \pm}\right) T \\
P \Delta \omega+c_{0} \frac{\partial \omega}{\partial x} \\
\Delta \Psi+\omega
\end{array}\right.
$$

It can be easily shown that the problem $L_{ \pm} u=0$ has only zero solution. Therefore the operator $L$ is normally solvable with a finite-dimensional kernel [21]. It remains to study 
solvability for the problem $L(u, c)=(f, g, h)$ where $f, g, h \in C^{(\delta)}(\bar{\Omega})$. We will solve each equation one after another.

The second equation is

$$
P \Delta \omega+c_{0} \frac{\partial \omega}{\partial x}=g,\left.\quad \omega\right|_{\partial \Omega}=0
$$

The corresponding operator is a Fredholm operator with the index zero. Moreover it does not have the zero eigenvalue. Thus the solvability conditions are satisfied for all $g \in C^{(\delta)}(\bar{\Omega})$ and there exists a solution $\omega \in C^{(2+\delta)}(\bar{\Omega})$.

The third equation

$$
\Delta \Psi=-\omega+h,\left.\quad \Psi\right|_{\partial \Omega}=0
$$

is solvable for any $h \in C^{(\delta)}(\bar{\Omega})$, and there exists a unique solution $\Psi \in C^{(2+\delta)}(\bar{\Omega})$.

Finally, we study the solvability of the problem

$$
\kappa \Delta T+c_{0} \frac{\partial T}{\partial x}+F^{\prime}(\theta) T=\left(\frac{\partial \Psi}{\partial z}-c\right) \theta^{\prime}(x)+f,\left.\quad \frac{\partial T}{\partial z}\right|_{\partial \Omega}=0 .
$$

The operator

$$
B u=\kappa \Delta T+c_{0} \frac{\partial T}{\partial x}+F^{\prime}(\theta) T
$$

acting from the space $C^{(2+\delta)}(\bar{\Omega})$ with the homogeneous Neumann boundary condition to the space $C^{(\delta)}(\bar{\Omega})$ satisfies the Fredholm property, it has the zero index, and a simple zero eigenvalue. The equation

$$
B u=f
$$

is solvable if and only if

$$
\int_{\Omega} f v d x=0
$$

where $v$ is a solution of the homogeneous formally adjoint equation

$$
\kappa \Delta T-c_{0} \frac{\partial T}{\partial x}+F^{\prime}(\theta) T=0,\left.\quad \frac{\partial T}{\partial y}\right|_{\partial \Omega}=0
$$

Its solution is unique up to a constant factor, and positive [14, 20]. Therefore for any $g \in C^{(\delta)}(\bar{\Omega})$ there exists a solution $(u, c)$ of the equation

$$
B u=g-c \theta^{\prime}(x) .
$$

This proves the solvability of problem (4.22).

Thus the operator $L: E_{\Theta} \times \mathbb{R}$ is invertible. This proves the applicability of the implicit function theorem and Theorem 1.1 in the bistable case. 
4.2. Monostable case. We consider now the monostable case which provides different properties of solutions for the stationary problem. We suppose that the wave speed is fixed, $c=c^{*}$, where $c^{*}$ is some value such that $c^{*}>2 \sqrt{\kappa F^{\prime}\left(\theta_{+}\right)}$. We will also impose some additional condition on the width $l$ of the strip (see Remark 4.2).

We consider now the problem linearized with respect to $u$ about the solution $u_{0}=$ $(\theta(x), 0,0) \in C^{(2+\delta)}(\bar{\Omega})$ for $R=0$. Consider the operator $L: E \rightarrow Z$

$$
L u=\left\{\begin{array}{l}
\kappa \Delta T+c^{*} \frac{\partial T}{\partial x}-\frac{\partial \Psi}{\partial z} \theta^{\prime}+F^{\prime}(\theta) T \\
P \Delta \omega+c^{*} \frac{\partial \omega}{\partial x} \\
\Delta \Psi+\omega .
\end{array}\right.
$$

We study solvability of the problem $L u=(f, g, h)$ where $f, g, h \in C^{(\delta)}(\bar{\Omega})$. For this purpose consider the solvability of each equation separately.

The second equation

$$
P \Delta \omega+c^{*} \frac{\partial \omega}{\partial x}=g,\left.\quad \omega\right|_{\partial \Omega}=0
$$

is solvable for any $g \in C^{(\delta)}(\bar{\Omega})$, and there exists a unique solution $\omega \in C^{(2+\delta)}(\bar{\Omega})$.

Then the third equation $\Delta \Psi=-\omega+h,\left.\Psi\right|_{\partial \Omega}=0$, is solvable for any $h \in C^{(\delta)}(\bar{\Omega})$, and there exists a unique solution $\Psi \in C^{(2+\delta)}(\bar{\Omega})$.

Consider finally the problem

$$
\kappa \Delta T+c^{*} \frac{\partial T}{\partial x}+F^{\prime}(\theta) T=\frac{\partial \Psi}{\partial z} \theta^{\prime}(x)+f,\left.\quad \frac{\partial T}{\partial z}\right|_{\partial \Omega}=0 .
$$

The operator

$$
B T=\kappa \Delta T+c^{*} \frac{\partial T}{\partial x}+F^{\prime}(\theta) T
$$

is a Fredholm operator with a positive index [5]. We will show that the codimension of its image equals zero, that is, there are no solvability conditions.

The corresponding homogeneous adjoint problem is

$$
\kappa \Delta T-c^{*} \frac{\partial T}{\partial x}+F^{\prime}(\theta) T=0
$$

with the following boundary conditions

$$
z=0, \quad l: \frac{\partial T}{\partial z}=0 .
$$

Furthermore we notice that

$$
F^{\prime}\left(\theta_{+}\right)=\lim _{x \rightarrow+\infty} F^{\prime}(\theta)>0, \quad c^{*}>0 .
$$


Multiplying equation (4.32) by $\cos (k z)$ and integrating over $z$, we obtain for the function

$$
u(x)=\int_{0}^{l} T(x, z) \cos (k z) d z, \quad k=\frac{2 \pi m}{l}, m \in R,
$$

the following equation:

$$
\kappa u^{\prime \prime}-c^{*} u^{\prime}+F^{\prime}(\theta) u=\kappa k^{2} u .
$$

It can be verified that it does not have nonzero bounded solutions (see Lemma 4.1 below). Hence the codimension of the image of the operator $B$ equals zero, and problem (4.30) is solvable for any right-hand side. Therefore we can apply the implicit function theorem. It proves Theorem 1.1 in the monostable case.

LEMMA 4.1. Let $u_{0}(x)$ be a bounded positive solution of the equation

$$
u^{\prime \prime}+c u^{\prime}+b(x) u=0,
$$

where $c>0, b_{+}=\lim _{x \rightarrow+\infty} b(x)>0, b_{-}=\lim _{x \rightarrow-\infty} b(x)<0$, and $c^{2}>4 b_{+}$. Then the equation

$$
u^{\prime \prime}-c u^{\prime}+b(x) u=\mu u
$$

does not have nonzero solutions for positive $\mu \neq b_{+}$.

Proof. Solution $u(x)$ of (4.38) behaves at $+\infty$ as $\exp (\lambda x)$ with

$$
\lambda=\frac{c}{2} \pm \sqrt{\frac{c^{2}}{4}-b_{+}+\mu} .
$$

If $\mu<b_{+}$, it is not bounded.

Suppose that $\mu>b_{+}$. Then the eigenvalue problem (4.38) has an the eigenvalue $\mu$ located to the right of the essential spectrum given by the expression

$$
\sigma_{\mathrm{ess}}=-\xi^{2}-c i \xi+b_{ \pm}, \quad \xi \in R .
$$

Therefore there exists a simple positive eigenvalue $\mu_{0} \geq \mu$ such that the corresponding eigenfunction is positive [14]. This eigenvalue is principal. Consequently, $\mu_{0}$ is also the principal eigenvalue of the problem

$$
u^{\prime \prime}+c u^{\prime}+b(x) u=\mu u
$$

and the corresponding eigenfunction $u_{1}(x)$ is also positive. We have for $x \rightarrow \infty$,

$$
u_{1}(x) \sim e^{\lambda_{1} x}, \quad \lambda_{1}=-\frac{c}{2}-\sqrt{\frac{c^{2}}{4}-b_{+}+\mu_{0}} .
$$

Consider the functions

$$
v_{0}(x)=u_{0}(x) \exp (\sigma x), \quad v_{1}(x)=u_{1}(x) \exp (\sigma x) .
$$


They satisfy the equations

$$
\begin{aligned}
& v^{\prime \prime}+(c-2 \sigma) v^{\prime}+\left(\sigma^{2}-c \sigma+b(x)\right) v=0, \\
& v^{\prime \prime}+(c-2 \sigma) v^{\prime}+\left(\sigma^{2}-c \sigma+b(x)\right) v=\mu_{0} v,
\end{aligned}
$$

respectively.

We take the value of $\sigma$ such that

$$
\frac{c}{2}-\sqrt{\frac{c^{2}}{4}-b_{+}}<\sigma<\frac{c}{2}+\sqrt{\frac{c^{2}}{4}-b_{+}} .
$$

Then the essential spectrum of the operator corresponding to the left-hand side of (4.45) lies in the left half-plane. On the other hand, $v_{1}$ is a bounded positive solution of (4.45). This contradicts the existence of the positive solution (bounded or unbounded) $v_{0}(x)$ of (4.44) [14].

Remark 4.2. We have proved that the codimension of the image of the operator $B$ in the monostable case equals zero under some additional conditions. Namely, we assume that $c^{*}>2 \sqrt{\kappa F^{\prime}\left(\theta_{+}\right)}$and $k^{2} \neq F^{\prime}\left(\theta_{+}\right) / \kappa$. The first assumption implies that the solution $\theta(x)$ is monotone. Nonmonotone solutions are unstable already for the scalar reaction-diffusion equation. Unstable solutions are not particularly interesting from the physical point of view.

The case of the equality, $c^{*}=2 \sqrt{\kappa F^{\prime}\left(\theta_{+}\right)}$is in some sense exceptional, and the situation here is not quite clear. On one hand, there is a monotone wave $\theta(x)$. On the other hand, we cannot move the essential spectrum to the left half-plane, and cannot conclude about the solvability conditions. We recall that it is the so-called KPP case that represents some peculiarities in wave behavior (see [16]).

The second restriction, which shows that there are some specific wavenumbers $k$ or, in the other words, values of the width $l$, is probably technical.

We have studied here the case of the Neumann boundary condition for the temperature. The case of the Dirichlet condition, $\left.T\right|_{\partial \Omega}=0$, is to some extent different but can also be studied by the same method [2]. The main difference is related to the computation of the index of the operator and to the solvability conditions.

\section{References}

[1] M. Bazile Jr., H. A. Nichols, J. A. Pojman, and V. Volpert, Effect of orientation on thermoset frontal polymerization, J. Polym. Sci. Part A: Polym. Chem. 40 (2002), no. 20, 3504-3508.

[2] M. Belk, Stabilité structurelle de solutions invariantes par translation. Application à des problèmes de réaction-diffusion avec convection, Ph.D. thesis, Université Claude Bernard Lyon 1, Villeurbanne, France, 2004.

[3] M. Belk, K. G. Kostarev, V. Volpert, and T. M. Yudina, Frontal photopolymerization with convection, J. Phys. Chem. B 107 (2003), no. 37, 10292-10298.

[4] G. Bowden, M. Garbey, V. M. Ilyashenko, et al., The effect of convection on a propagating front with a solid product: comparison of theory and experiments, J. Phys. Chem. B 101 (1997), 678-686.

[5] J. F. Collet and V. A. Volpert, Computation of the index of linear elliptic operators in unbounded cylinders, J. Funct. Anal. 164 (1999), no. 1, 34-59. 
[6] L. Debnath, Nonlinear Partial Differential Equations for Scientists and Engineers, 2nd ed., Birkhäuser Boston, Massachusetts, 1997.

[7] M. Garbey, A. Taik, and V. Volpert, Linear stability analysis of reaction fronts in liquids, Quart. Appl. Math. 54 (1996), no. 2, 225-247.

[8] Influence of natural convection on stability of reaction fronts in liquids, Quart. Appl. Math. 56 (1998), no. 1, 1-35.

[9] B. McCaughey, J. A. Pojman, C. Simmons, and V. A. Volpert, The effect of convection on a propagating front with a liquid product: comparison of theory and experiments, Chaos 8 (1998), no. 2, 520-529.

[10] J. Pojman, S. Popwell, D. Fortenberry, Vit. Volpert, and Vl. Volpert, Nonlinear dynamics of SHS-like processes in polymer chemistry, Self-Propagating High-Temperature Synthesis of Materials (A. A. Borisov, L. T. De Luca, and A. G. Merzhanov, eds.), Taylor and Francis, New York, 2002, pp. 106-134.

[11] S. Smale, An infinite dimensional version of Sard's theorem, Amer. J. Math. 87 (1965), 861-866.

[12] R. Texier-Picard and V. A. Volpert, Problèmes de réaction-diffusion-convection dans des cylindres non bornés, C. R. Acad. Sci. Paris Sér. I Math. 333 (2001), no. 12, 1077-1082 (French).

[13] , Reaction-diffusion-convection problems in unbounded cylinders, Rev. Mat. Complut. 16 (2003), no. 1, 233-276.

[14] A. I. Volpert and V. A. Volpert, Spectrum of elliptic operators and stability of travelling waves, Asymptot. Anal. 23 (2000), no. 2, 111-134.

[15] _ Existence of multidimensional travelling waves and systems of waves, Comm. Partial Differential Equations 26 (2001), no. 3-4, 421-459.

[16] A. I. Volpert, V. A. Volpert, and V. A. Volpert, Traveling Wave Solutions of Parabolic Systems, Translations of Mathematical Monographs, vol. 140, American Mathematical Society, Rhode Island, 1994.

[17] V. Volpert, V. Volpert, M. Garbey, and J. Pojman, Instabilities of reaction fronts, Gas Phase Chemical Reaction Systems (J. Wolfrum, H.-P. Volpp, R. Rannacher, and J. Warnatz, eds.), Springer Series in Chemical Physics, vol. 61, Springer, Berlin, 1996, pp. 309-317.

[18] V. A. Volpert and A. I. Volpert, Wave trains described by monotone parabolic systems, Nonlinear World 3 (1996), no. 2, 159-181.

[19] Convective instability of reaction fronts: linear stability analysis, European J. Appl. Math. 9 (1998), no. 5, 507-525.

[20]_, Existence and stability of multidimensional travelling waves in the monostable case, Israel J. Math. 110 (1999), 269-292.

[21] V. A. Volpert, A. I. Volpert, and J. F. Collet, Topological degree for elliptic operators in unbounded cylinders, Adv. Differential Equations 4 (1999), no. 6, 777-812.

M. Belk: Laboratoire de Mathématiques Appliquées, UMR 5585 CNRS, Université Lyon 1, 69622 Villeurbanne, France

E-mail address: belk@maply.univ-lyon1.fr

B. Kazmierczak: Institute of Fundamental Technological Research, Świetokrzyska 21, 00-049 Warsaw, Poland

E-mail address: bkazmier@ippt.gov.pl

V. Volpert: Laboratoire de Mathématiques Appliquées, UMR 5585 CNRS, Université Lyon 1, 69622 Villeurbanne, France

E-mail address: volpert@maply.univ-lyon1.fr 


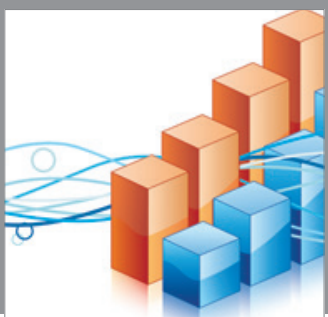

Advances in

Operations Research

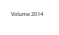

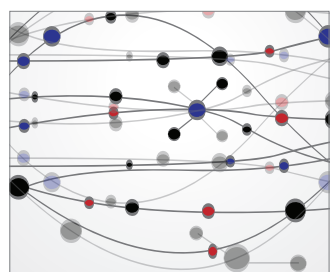

\section{The Scientific} World Journal
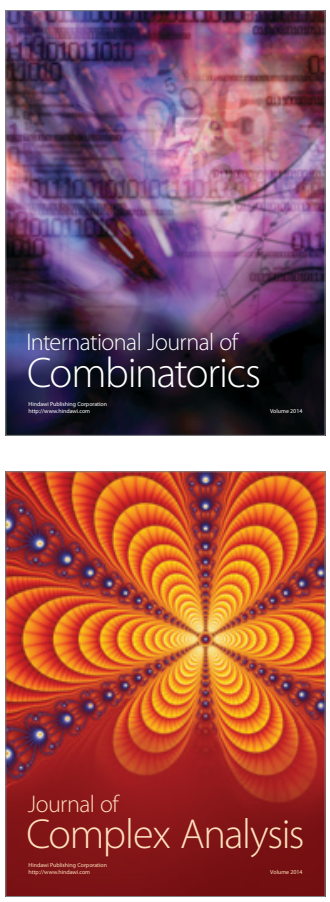

International Journal of

Mathematics and

Mathematical

Sciences
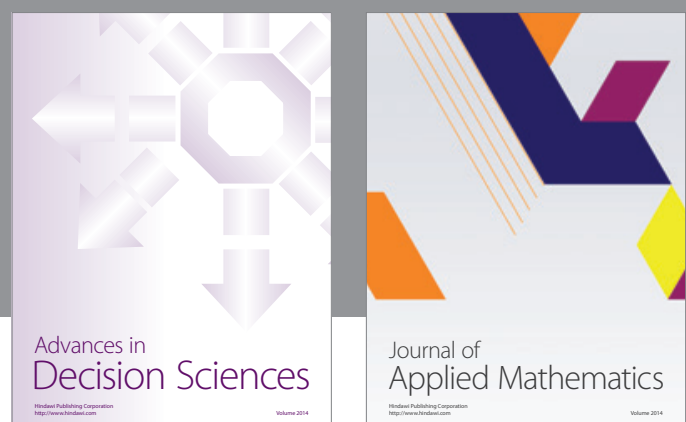

Journal of

Applied Mathematics
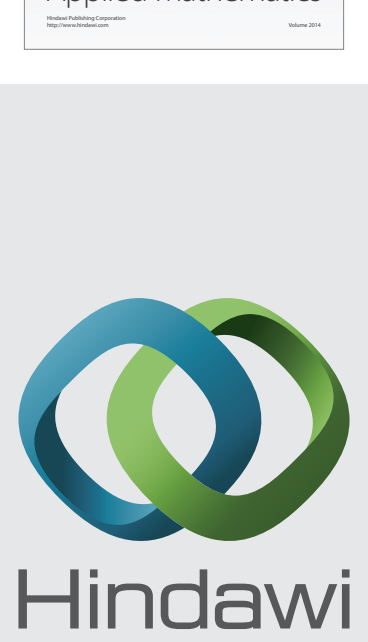

Submit your manuscripts at http://www.hindawi.com
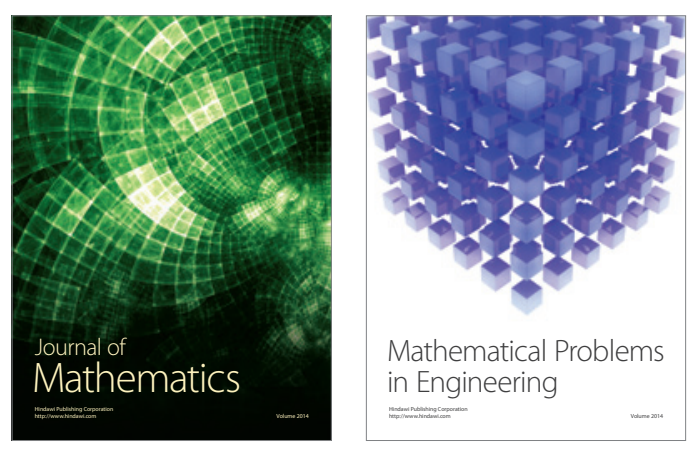

Mathematical Problems in Engineering
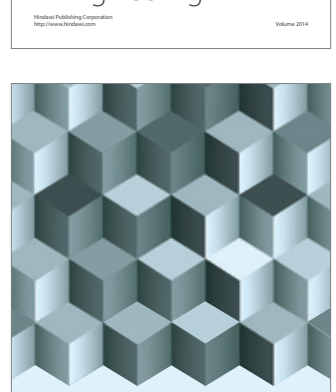

Journal of

Function Spaces
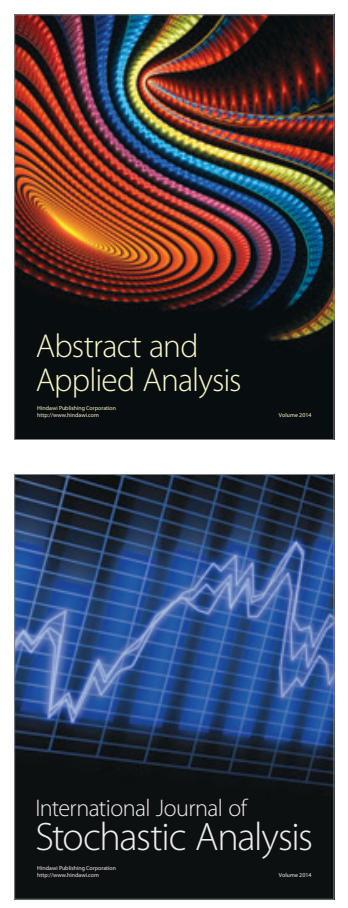

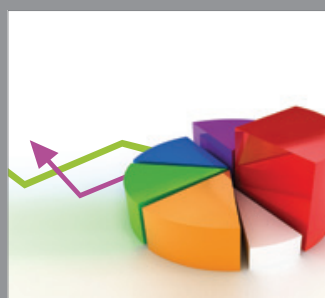

ournal of

Probability and Statistics

Promensencen
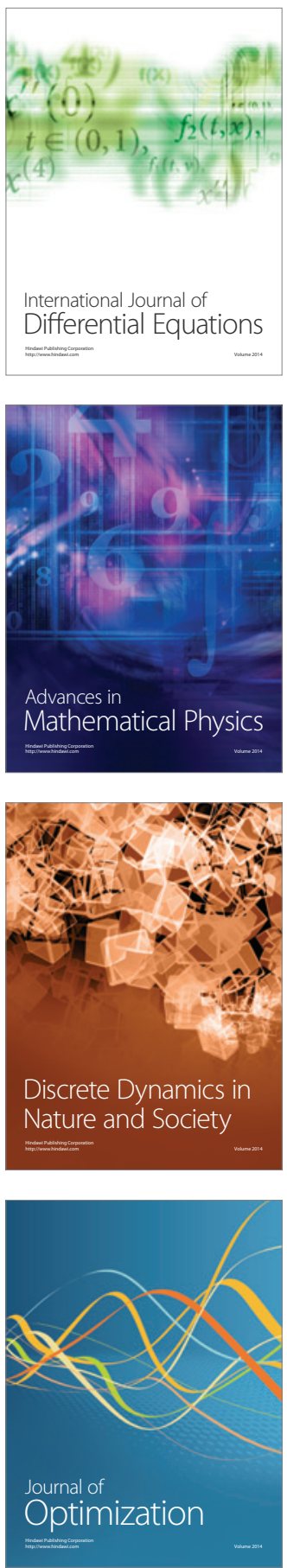